\title{
The Froth Thickens
}

\section{Frothing can arise in mixtures of two fully miscible liquids with nonlinear composition-dependent surface tensions.}

\author{
By Gerald Fuller and Vinny Chandran Suja
}

A s the physicist and author Sidney Perkowitz observes in his popular science book Universal Foam: From Cappuccino to the Cosmos, foams and froths impact almost every major branch of science [1]. For example, liquid foams-liquids that contain a suspension of gas bubbles-are an intrinsic part of many fire retardants and of many food products, such as beer [2]. These foams are stable, retaining their structure and resisting collapse into a liquid, which is beneficial for these systems. That stability is undesirable in foams generated-unintentionally-during lubrication, textile manufacturing, and paper production. Industry would like to avoid making these foams, but why the foams are so stable has, until now, remained a mystery. New experiments and models
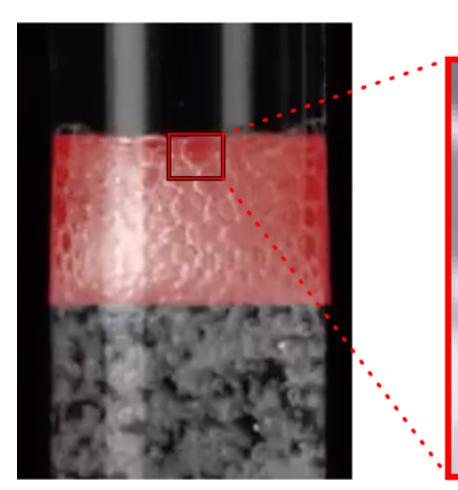

Lamella

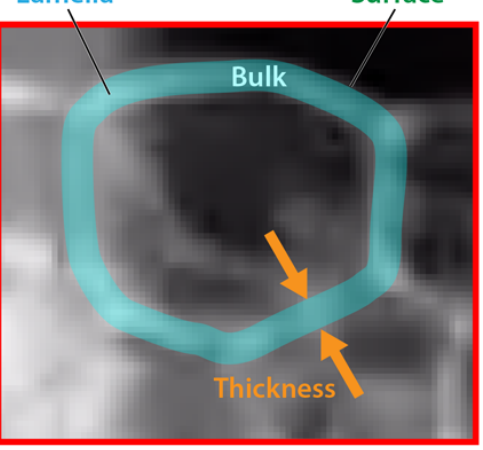

Figure 1: Bubble air through a fluid made of two miscible liquids, and a stable foam can form at the fluid's surface. This foam contains bubbles separated by films or lamellae (inset, blue). The lamellae vary in thickness across their length, introducing surface tension gradients around each bubble within the foam. These gradients inhibit lamella thinning and provide the stabilizing force for the foam.

Credit: H.-P. Tran/PSL University by Hoai-Phuong Tran, at PSL (Paris Sciences \& Letters) University, and colleagues now show that the stability of these foams comes from surface tension gradients that arise within the frothy liquid [3].

The bubbles that form in a single component liquid pop quickly, giving any foams a very short lifetime. Modifying the liquid's properties may prolong these lifetimes, allowing the formation of a stable foam. For example, increasing the viscosity of the liquid can retard the thinning of the thin liquid walls, known as lamellae, that exist between a foam's bubbles and inhibit foam collapse. Adding surface-active particles or surfactants can also lead to stable foams $[4,5]$. These particles sit on the liquid-air interfaces found on both sides of a lamella. Repulsive electrostatic interactions between particles on opposing interfaces prevent the fusing of the two liquid surfaces, halting foam collapse. For foams containing surfactants, collapse delay arises from the surface tension gradients that the surfactants induce across the length of a lamella. The gradients generate flows on the surface of each lamella, which move from regions of low to high surface tension. These surface flows on either side of the lamella oppose and slow the thinning of the lamellae. When the lamellae become very thin, certain surfactants can arrest further thinning and film rupture, similarly through long-range repulsive, electrostatic interactions.

Still, researchers don't yet understand froth formation in surfactant-free binary liquids where the two fluids are fully miscible. Such mixtures include most petroleum products, which consist of a distribution of miscible components. The experiments and theory offered by Tran and colleagues provide a framework for explaining how foams form and stabilize in such mixtures.

To explore how a foam forms in a binary, miscible liquid, the 


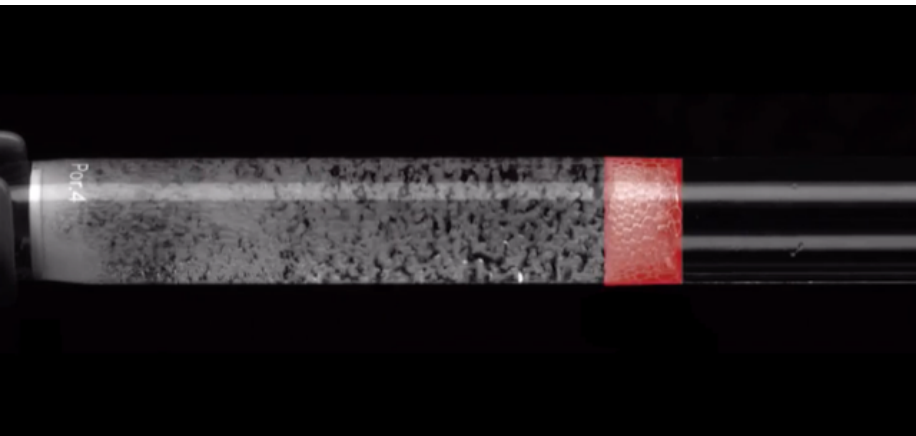

Video 1: Bubbling air through a mixture of two liquids results in the formation of a foam whose height $H$ depends on the surface tension of the two liquids.

Credit: H.-P. Tran/PSL University

researchers performed experiments on mixtures of toluene and alkanes and on mixtures of two different alcohols. The fluids were combined in a glass cylinder and air was then bubbled through the bottom of the cylinder. The team measured the height of the resulting foam (Fig. 1 and Video 1). Interestingly, they only observed foams in binary fluids where the surface tension of the fluid had a nonlinear dependence on the concentration of each of the liquids in the solution.

To explain their observations, Tran and his colleagues developed a theory that accounts for a surface tension that depends on lamella thickness. In the theory, the thickness dependence is explained as follows: As a lamella thins, there is an increase in the lamella surface-area-to-volume ratio and a fall in the surface concentration of the low-surface-tension liquid, which is more concentrated on a lamella's surface than in its bulk. This change causes an increase in the lamella's overall surface tension. To maintain the equilibrium surface-to-bulk concentration, low-surface-tension liquid from the bulk then rapidly diffuses to the surface, lowering the overall surface tension to its original value. This process continues as long as the bulk concentration remains near its original value. But when the bulk concentration falls below a critical value, which happens when the lamella gets too thin, the surface concentration of the low-surface-tension liquid drops, and the lamella's overall surface tension rises. If the lamella thickness is not constant across its length, this behavior can result in surface-tension variations across a lamella. Those variations can lead to fluid flows that stabilize the foam in a manner similar to those seen in surfactant-containing fluids.

A necessary condition for thickness-dependent surface tension is an excess surface concentration of the low-surface-tension liquid. That excess usually manifests as a mixture whose surface tension varies nonlinearly as the concentration of one liquid changes. If, instead, that variation is linear, the mixture will not exhibit any surface excess and should not froth. That finding is precisely what Tran and colleagues see in their experiments.

These new findings provide a mechanism by which froths can stabilize and raise new questions about miscible liquid systems. Petrochemical and lubricant industries routinely utilize miscible binary liquid mixtures in situations where froth formation needs to be tightly controlled. The theory Tran and colleagues propose indicates that frothing can be mitigated by careful choice of the liquids. In situations where this choice is unfeasible, the critical thickness predicted by the theory could help identify what size of additives to use to inhibit frothing: to accelerate foam collapse, the additives needs to be wide enough to span a lamella [6].

The results also raise questions about the dynamics of the fluid inside a lamella in the presence of thickness-dependent surface tension changes. How do changes in the lamellae thickness seen in this mechanism compare to those seen in other stabilization mechanisms $[4,5,7,8]$ ? Can thickness-dependent changes in surface tension drive dynamic phenomena, such as the spontaneous formation and collapse of "dimples" in the lamella, which are known to occur for evaporation-driven foam stabilization $[8,9]$ ? Answering these questions will provide new insights into this foamy field: The future is bubbling with excitement.

Gerald Fuller: Department of Engineering, Stanford University, Stanford, California USA

Vinny Chandran Suja: Department of Engineering, Stanford University, Stanford, California USA

\section{REFERENCES}

1. S. Perkowitz, Universal Foam: From Cappuccino to the Cosmos (Walker \& Co., New York, 2000).

2. R. J. Pugh, Bubble and Foam Chemistry (Cambridge University 
Press, Cambridge, 2016).

3. H. -P. Tran et al., "Understanding frothing of liquid mixtures: A surfactantlike effect at the origin of enhanced liquid film lifetimes," Phys. Rev. Lett. 125, 178002 (2020).

4. D. Langevin, "Bubble coalescence in pure liquids and in surfactant solutions," Curr. Opin. Colloid Interface Sci. 20, 92 (2015).

5. S. E. Friberg, "Foams from non-aqueous systems," Curr. Opin. Colloid Interface Sci. 15, 359 (2010).

6. V. Chandran Suja et al., "Foam stability in filtered lubricants containing antifoams," J. Colloid Interface Sci. 567 (2020).

7. S. Ross and G. Nishioka, "Foaminess of binary and ternary solutions," J. Phys. Chem. 79, 1561 (1975).

8. V. Chandran Suja et al., "Evaporation-induced foam stabilization in lubricating oils," Proc. Natl. Acad. Sci. U. S. A. 115, 7919 (2018).

9. O. D. Velev et al., "Spontaneous cyclic dimpling in emulsion films due to surfactant mass transfer between the phases," J. Colloid Interface Sci. 159, 497 (1993). 